\title{
License Plate Recognition for Indian Number Plate: A Review
}

\author{
Nandan More \\ Department of Computer Engineering \\ Flora Institute of Technology, \\ Pune, India.
}

\author{
Bharat Tidke \\ Department of Computer Engineering \\ Flora Institute of Technology, \\ Pune, India.
}

\begin{abstract}
Traffic security and its management controls and supervised the number of vehicles. The traffic management system broadly used license plate recognition for them whose owner has dumped the traffic laws or to find the stolen vehicles. In most of the traffic related application like stolen vehicles, road traffic monitoring airport gate monitoring, speed monitoring, and automatic parking lots access control, for all this purpose the key technique are used which are vehicle license plate detection and recognition. It is basically the skill of automatically extract and recognition of the vehicle license number plate character from the captured image. The method of number plate recognition faces the problem of feature selection process. The present methods of number plate recognition system only focus on the local, global and neural network process of feature extraction and process of detection. The detection ratio of number plate recognition was improved by the optimized feature selection process. Here in this paper novel method for license plate recognition is proposed, which is based on wavelet transform function as well as comparing the proposed methodology with correlation based method for the detection of number plate.
\end{abstract}

Index Terms: Feature selection, RBF Neural Network, vehicle license plate detection, wavelet transforms.

\section{INTRODUCTION}

License plate recognition method which is an image technology implemented for identifying vehicle number plate[4]. The technology is gaining popularity in security and traffic facilities and goal of LPR was to build an application capable of automatically recording license plate numbers of passing vehicles traveling down a roadway. These days, the license plate recognition is broadly employed in traffic laws or to find stolen vehicles. In most of the traffic related application like stolen vehicles, road traffic monitoring airport gate monitoring, speed monitoring, and automatic parking lots access control, for all this purpose the key technique are used which are vehicle license plate detection and recognition[14].

\section{BACKGROUND THEORY}

In order to recognize license plate, remains the principle factor in identifying vehicle despite the fact that it can be deliberated altered in fraud situation. Technique has achieved much greater interest during the last few decades along with the upgrading of the digital cameras and increased in computational capacity. The reason of this automatic license plate recognition system is to impact illicit drug trafficking on the regional stability of the metro city in the particular combination with other present destabilizing factors. Basically it is the ability to automatically extract and recognition of the vehicle license number plate's character from the capture image. It is fundamentally subsist of frame grabber or camera which has the capability to grab an image, locate the location of the number in the figure and take out the character of an instrument of character recognition to convert the pixel into easily readable character. The method can also be used in the highly sensitive areas like military zones or area above top government head offices. By using ANPR system installed on the highway the recognition of the stolen vehicle can be done.

\section{PREPROCESSING}

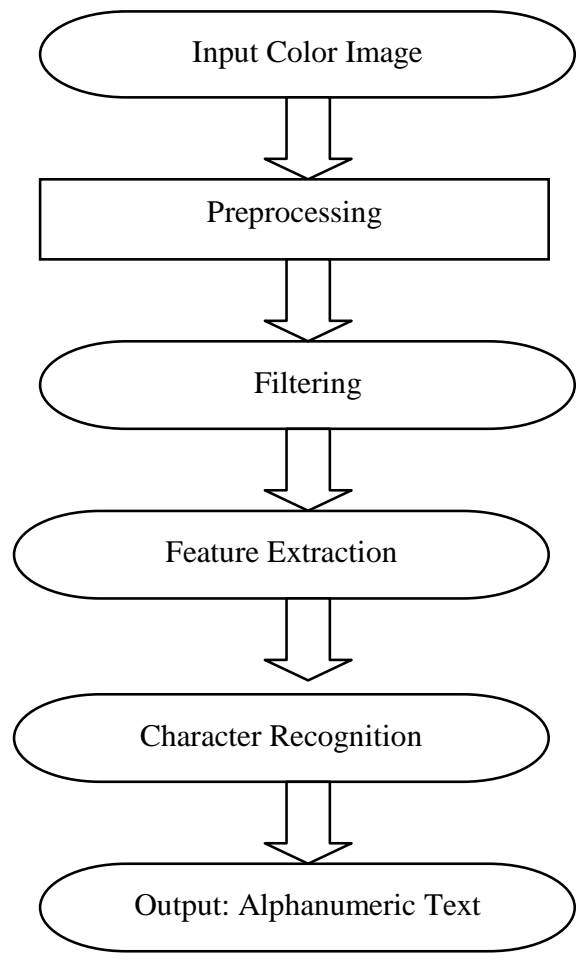

Fig.1: System Structure

1. Detection and capturing of vehicle image

2. Detection and extraction of number plate in an image

3. Use image segmentation method to get the individual character and Optical Character Recognition 
(OCR) to recognize the individual character with the help of database stored for each and every alphanumeric character [3].

Using four parts the LPRS (License Plate Recognition System) is made up in which sub process present in the each process (see figure 3.1 )

a. The inseparable and integral part of the system is LP image Location-License Plate Location because it locates the plate that encloses the license plate numbers. There are enormous data in the image that's why on the edges of the license plate only the entire concepts are depending. The major factor is the extraction of multiple license plates from an image with a complex background. To extract the license plate different processes is performed. The segmentation parts get the output from the extractor.

b. Character Separation also called as Character Segmentation-License plate segmentation. From a picture a license plate images are extracted and after that individual character is find out for the reorganization. In the license plate characters segmentation first license plate is first converted into binary image after that for obtaining the characters separately, the characters are divide into segments to essential parts [4]. In arrange to outline the individual's characters, license plate segmentation is useful. Character isolation which is referred to as License Plate Segmentation takes the region of interest and attempts split it into individual characters.

c. The last step of the LPR system is Character Recognition-License. It is the main part of the recognition process which decides the accuracy and recognition rate of the system. It involves about to recognize the characters of the license plate characters and numbers [4] [5]. License plate characters are normalized before the recognition. Normalization is to improve the characters into a block containing no added white spaces (pixels) in all the four sides of the characters. In this stage license plate character images have to be recognizing which is taken out from the license plate image. It is actually the process of the character recognition of the license plate characters which is generally find out through Neural Network, Hough Transformation, Radial Basic Function and Template matching.

d. In this survey on various research paper about the number plate recognition. The Indian number plates don't have any particular standard number plate format (myriad). But in current scenario Government of India issues standard number plate and standard templates of characters and number's. Various agencies working in the field of Indian license plate recognition system in order to maintain traffic problem and controlling crime.

\section{RELATED WORK}

In the literature survey, survey has been taken on the license plate detection. On the basis of survey here some techniques has been described such as license plate detection using template matching and RBF Neural Network. The technology is gaining popularity in security and traffic facilities.

\subsection{License Plate Detection using RBFNN}

In this technique with the help of text-line construction and multilevel RBFNN a license plate is detected. According to Baoming Shan [2] the text line construction result and the vehicle license plate character arrangement characteristics the license plate location is decided. And to create more precise license plate localization a locally best adaptive binarization is exploiting. After completion of license plate localization, to segment character and extract the statistic feature the method of vertical projection information with prior knowledge is proposed. After that the multilevel classification RBF neural network is used to identify with feature vectors as input. Due to this identification result is reliable and satisfied by the accuracy requirement of the Intelligent Transportation Systems. Improving the accuracy of the characters recognition is done by using multilevel classification of RBFNN

The Radial Basis Function Neural Network is used by many authors Baoming Shan [2] Nureddin A. Abulgasem, Dzulkili Mohamad [6]. It is used to identify and detect the license plate, image preprocessing process such as filling and smoothing for improving the quality of the image, image dilation, edge detection, filtering. Here in preprocessing gray scale conversion of the image is performed and then with the help of morphological detector and sobel edge detector the gray scale image converted into black and white image "flood fill algorithms" [13] is used and after finishing of all this steps, this preprocessed image used for LP detection. In this process a maximum and minimum threshold values are used and on the basis of this values classification is done. Objects those values satisfy the minimum and maximum threshold values are classified as "Plate" and "No Plate" categories. For LP detection RBFNN method is used. The RBFNN is trained using the objects which are classified in a "Plate" category. The RBFNN is used with feature extraction process for detection of the character where original feature are extracted and nourish into $\mathrm{NN}$ to classify the characters and the found 99\% character segmentation rate. The character segmentation identification from other images may leads to mistaken license plate detection [6].

To overcome above problem Sobel edge detector, vertical and horizontal projection and mathematical morphology (MM) operation used for LP region and it is a new technique for license plate recognition system also it eliminates the component whose height does not match with the characters from the plate and provides the segmented characters. With the help of this it finds the exact height of the characters. The system may fail if the texture of the plate is not clear and the License plate region is short of the threshold of projection operation.

Based on the sharing features of a diversity of license plates (LP), [9] the vertical edge was first detected by Sobel edge detector. Then, some approaches were accepted to eliminate the invalid edge concerning the characteristics of edge grayscale jump and edge density, so that the areas having features of LP were potted. Next, by horizontal and vertical projections and mathematical morphology (MM) operation, the LP region was searched. Then, color-reversing judgment was behavioral by color analysis, and binarization was done based on core region in LP. Afterward, characters were segmented by means of prior knowledge and connected components analysis, and character recognition was conducted based on radial basis function (RBF) neural network. With plentiful samples established in dark hours and daytime under real conditions, the experiment point out that it 
is practicable to adopt this algorithm in license plate recognition system (LPRS) to attain correctness.

The diversities of License Plates (LP) are concerned in the License Plate Identification System. For recognition process the LVQ (Learning Vector Quantization) and image correlation is used. To increase the probability of recognition of the right variable a hybrid method uses the image correlation and NN. That's why hybrid method is better for identification. All unwanted regions are removed in the character segmentation process it means it segment the $100 \%$ characters. But if the correlation value is greater or equal than $1 / 2$ then it believe the character as a correct where as character may be wrong and using neural network the correct character is get. But it does not accept character recognize using the neural network but accept using the correlation [8].

\subsection{License Plate Detection using Template Matching}

ALPRSIV (Automatic license plate recognition system for Indian vehicles) is implemented using image processing and machine vision engage in the license plate recognition field. It comprises algorithms which make certain suitable character segmentation process, plate normalization process, identification and normalization. All this methods are achieving invariance of systems towards image skew homographs and a variety of plate condition. The authors gave the innovative approach for License plate recognition based on Neural Network. To recognize the number plate the neural network chip is used. The chip composed of two modules video image processing module with neural network module using equalized image processing algorithm and network classification algorithm. The CMIK system performs better in stability than software system, because most of the circuit was integrated in the FPGA, and the bottom hardware finished much amount work of recognition.

The license plate recognition system as uses an algorithm novel adaptive image segmentation technique (Sliding Concentric Windows-SCW) and connected component analysis in conjunction with a character recognition Neural Network. The algorithm was tested with particular natural scene gray level vehicle images of different backgrounds and ambient illumination.

The author presents a new smart and simple algorithm as well as LPR works in different parts: plate region extraction, character segmentation and recognition. The recognition process is done with the help of template matching. The detection of the plate is achieved with the higher accuracy. The similarity between some characters decreases the recognition rate. [5]. In the paper [2], they attempt to provide an improve and complete sight of the research work made in the area of LP detection and recognition and the methods working in rising a License plate Recognition (LPR) system. In this analysis contrast the License Plate Recognition methods based on dissimilar criterion like character segmentation.

In paper [1] Automatic license plate recognition system is used many applications such as: parking entrance, highway toll fee collection, red light enforcement etc. in this paper learn automatic license plate recognition system by presenting in a dissimilar technique. First, Mean Value Algorithm is used in order to locate the plate. Then the characters of the plate are segmented. Finally, the characters are documented by using a correlation method.
Serkan ozbay and Ergun Ercelebi presents a license plate detection algorithm from multifaceted background based on histogramming and mathematical morphology [3]. The projected algorithm consists of two main modules: license plate region's rough detection and license plate exactly location. The previous characterized by vertical gradients detection extracts candidate areas from an input image, while the latter conceptualized in terms of mathematical morphology aspires to locate the license plate quick and precisely.

In the paper [4] presents Automatic Number Plate Extraction and Character Segmentation for Indian vehicles. India, number plate models are not followed firmly. Characters on plate are in different Indian languages, as well as in English Due to difference in the representation of number plates, vehicle number plate extraction and character segmentation is hard. They work on plates that contain English characters. The projected algorithm consists of three major parts: Preprocessing of Image, Extraction of plate region and Segmentation of characters. For preprocessing of the image, histogram equalization has been applied. For extracting the Plate region, edge detection algorithm and various morphological operations are used. Character segmentation is done by using connected component and bounding box method. In [10] a license plate recognition system based on neural networks was planned and developed. The system used a neural-network chip to recognize license plates. The chip combined video image processing module with neural network module by using equalized image processing algorithm and network classification algorithm. A set of interface circuit was developed for implementing licenseplate-number recognition. Experiment results show the systems can assurance a very low error rate at a satisfactory recognition time.

\section{CONCLUSION}

The procedure of vehicle number plate recognition requires a very high degree of correctness when in this are working on a very full of activity road or parking which may not be possible manually as a human being tends to get exhausted due to dull nature of the job and they cannot stay path of the vehicles when there are numerous vehicles are passing in a very short time .To conquer this problem, many efforts have been made by the researchers across the globe for last many years. A alike effort has been made in this dissertation to expand an accurate and automatic number plate recognition system. In this dissertation in this intend a license plate recognition technique for the improvement of the recognition rate and recognition time for recognition of the number and the character of the vehicle license plate. It is projected a new technique of Neural Network for Vehicle license plate recognition. The Neural Network generates less recognition times and improves the recognition made in this dissertation to develop an accurate and automatic number plate recognition system. In this dissertation in this propose a license plate recognition technique for the improvement of the recognition rate and recognition time for recognition of the number and the character of the vehicle license plate. It is proposed a new technique of Neural Network for Vehicle license plate recognition. The Neural Network produces less recognition times and improves the recognition time of the license recognition system. System work demonstrates better performance as compare to the correlation method which is one of the efficient techniques for matching. Therefore, the standard divergence error reduces which comes 
from the data lost during the preprocessing in the recognition process.

In this system required to minimize the processing time. And pattern should be segmented correctly. System is able to minimize the processing time and if recognition time is reduce then we can do recognition and detection process with Face Detection simultaneously.

\section{REFERENCES}

[1] D. Renuka Devi \& D. Kanagapushpava Ui. 2011. Automatic License Plate Recognition, pp. 75-78 IEEE.

[2] Boaming Shan. February 2011. Vehicle License Plate Recognition Based on Text-line Construction and Multilevel RBF Neural Network. Journal of computer science Vol. no. 6pp. 246-253.

[3] Serkan ozbay and Ergun Ercelebi. 2005. Automatic Vehicle Identification by Plate Recognition. World Academy of Science, Engineering and Technology 9 pp. 222-225.

[4] Chetan Sharma and Amandeep Kaur July-December 2011. Indian Vehicle License plate Extraction and Segmentation. International Journal of Computer Science and Communication Vol. No.2,pp. 593-599.

[5] Serkan Ozbay, and Ergun Ercelebi. Sep 2007. Automatic Vehicle Identification by Plate Recognition. World Academy of Science, Engineering and Technology, pp 778-781.

[6] Nureddin A. Abulgasem, Dzulkili Mohamad, Siti Zaiton Mohamad Hashim. 2011.Automatic License Plate Detection and Recognition Using Radial Basis Function Neural Network. Indian Journal of Computer Vision and Applications Vol. I.No. I pp. 15-23.

[7] Shishir Kumar, Shashank Agarwal \& Kumar Saurabh. July-December 2008.License Plate Recognition System for Indian Vehicles. International Journal of Information Technology and Knowledge Management, Volume 1, No.2, pp. 311-325
[8] K. Yilmaz. 2011. A smart Hybrid License Plate Recognition System Based on Image Processing using Neural Network and Image Correlation. pp. 148-153 IEEE.

[9] Bo Li, Zhi-yuang, Jian-zhong, Zhou and Huali Dong. 2008. An Algorithm for License Plate Recognition Using Radial Basis Function Neural Network. International Symposium on Computer Science and Computational Technology pp. 569-572.

[10] Yi Qing Liu and Dong Wei, Ning Zhang and Min Zhe Zhao. June 2011. Vehicle-License-Plate Recognition Based on Neural Networks. Proceedings of IEEE International Conference on Information and Automation Shenzhen, pp 363-366, China

[11] S. Hamidreza Kasaei, S. Mohammadreza Kasaei, S. Alireza Kasaei. April 2010. New Morphology-Based Method for Robust Iranian Car Plate Detection and Recognition. International Journal of Computer Theory and Engineering, Vol.No.2, pp 268-268.

[12] C.N. Anagnostopoulos, I. Anagnostopoulos,V. Loumos, and E. Kayafas August 29,2005. A license plate recognition algorithm for Intelligent Transportation System applications. TITS-05-08-0094

[13] Sourav Roy,Amitava Choudhury,Joydeep Mukherjee March2013. An Approach towords Detection of Indian Number Plate from Vehicle. International Journal of Innovative Technology and Exploring Engineering (IJITEE)ISSN:2278-3075,Volume-2,Issue-4.

[14] Jitendra Sharma, Amit Mishra,Khushboo Saxena, Shiv Kumar Feb 2014. A Hybrid Technique for License Plate Recognition Based on Feature Selection of Wavelet Transform and Artificial Neural Network. International Conference on Reliability,Optimization and Information Technology ICROIT,2014,India. 